\title{
Mentally healthy days and days out of role associated with psychological distress: Relating the General health Questionnaire with functioning
}

\author{
A. Lundin ${ }^{1,2}$, C. Hogstedt ${ }^{3}$. \\ ${ }_{1}^{1}$ Centre for Epidemiology and Community Medicine, Stockholm County Council, Stockholm, Sweden. \\ 2 Karolinska Institutet, Department of Public Health Sciences, Karolinska Institutet, Stockholm, Sweden. \\ ${ }^{3}$ Uppsala University, Department of Medical Sciences, Occupational and Environmental Medicine, Uppsala, Sweden.
}

Objectives: To compare the twelve-item version of the General Health Questionnaire, a common measure of distress, to global measures of functional limitation.

Background: Epidemiological surveys have shown that mental disorders are associated but not synonymous with functional impairment. Many public health surveys do not include diagnositic schemes but rely on more general distress scales such as the GHQ-12 (1). Distress scales are good indicators of overall mental health in communities, covering also those otherwise missed as subclinical cases and without having to consider effects of comorbidity (2). There are however few studies which have examined the association between distress and global measures of functional limitation. The aim of this study was to compare the GHQ-12 with measures of days with metal illness and days out of role.

Materials and Methods: We used a general population survey of 18-84 year olds in Sweden in $2012(n=9,089)$ containing GHQ-12 and the CDC Core Healthy Days (3) Associations between GHQ-12 and number of 30 past days when 1) mental health was not good, and 2) days when poor physical or mental health limited usual activities (out of role), were tested with negative binomial regression. Confounding from poor physical health was checked with days in the past 30 past days when physical health was not good, as well as age and sex.
Results and conclusion: Mean ill mental days and activity limitations days were 5.2 and 4.6. Mean ill physical health was 7.1. All measures of functional limitations increased with GHQ12 symptom score (distress, Figure). GHQ-12 predicted both days with poor mental health and functional limitations holding physical health constant (expected log count days per GHQ-12 step $=0.22(p<0.001)$ and $0.24(p<0.001)$, Table $)$. GHQ-12 has a strong association with functioning which is independent of physical functioning.

Table. Estimated negative binomial regression coefficients (log rate)

\begin{tabular}{|c|c|c|c|c|c|c|}
\hline & \multicolumn{2}{|c|}{ Physically unhealthy days } & \multicolumn{2}{|c|}{ Mentally unhealthy days } & \multicolumn{2}{|c|}{ Out of role days } \\
\hline & B & $95 \% \mathrm{Cl}$ & $\mathrm{B}$ & $95 \% \mathrm{Cl}$ & $B$ & $95 \% \mathrm{Cl}$ \\
\hline \multicolumn{7}{|l|}{ Model 1} \\
\hline GHQ-12 & 0.14 & $0.12-0.15$ & 0.25 & $0.23-0.27$ & 0.24 & $0.21-0.26$ \\
\hline Age & 0.01 & $0.01-0.01$ & -0.002 & $-0.005-0.0009$ & 0.02 & $0.02-0.02$ \\
\hline Sex & 0.17 & $0.10-0.24$ & 0.24 & $0.16-0.32$ & 0.14 & $0.04-0.25$ \\
\hline Dispersion & 2.90 & $2.80-3.01$ & 3.43 & $3.30-3.57$ & 6.08 & $5.83-6.35$ \\
\hline \multicolumn{7}{|l|}{ Model 2} \\
\hline GHQ-12 & 0.06 & $0.04-0.07$ & 0.22 & $0.20-0.24$ & 0.20 & $0.18-0.21$ \\
\hline Age & 0.01 & $0.01-0.02$ & -0.01 & $-0.02--0.01$ & 0.003 & $0.001-0.005$ \\
\hline Sex & 0.14 & $0.07-0.21$ & 0.22 & $0.15-0.30$ & 0.09 & $0.004-0.18$ \\
\hline Physically unhealthy & NA & NA & 0.07 & $0.06-0.07$ & 0.11 & $0.11-0.12$ \\
\hline Mental unhealthy & 0.05 & $0.05-0.06$ & NA & NA & NA & NA \\
\hline Dispersion & 2.66 & $2.57-2.76$ & 2.75 & $2.64-2.87$ & 3.75 & $3.57-3.93$ \\
\hline
\end{tabular}

Note: $\mathrm{B}=$ beta coefficent $(\log$ rate), $95 \% \mathrm{Cl}=95 \%$ Confidence intervals. Dispersion $=$ dispersion parameter of the response variable. $\mathrm{n}=9586$

\section{References:}

1. Goldberg et al. The validity of two versions of the GHQ in the WHO study of mental illness in general health care. Psychological medicine . $1997,27.191-197$

2. Korten $A$ and Henderson S, The Australian national Survey of mental Health and Well-Being. Common psychological symptoms and disablement. British Journal of Psychiatry 2000, 177. 325-330

3. Moriarty DG, Zack MM Kobau R. The Centers for Disease Control and Prevention's Healthy Days Measures - Population tracking of perceived physical and mental health over time. Health and Quality of Life Outcomes 2003,

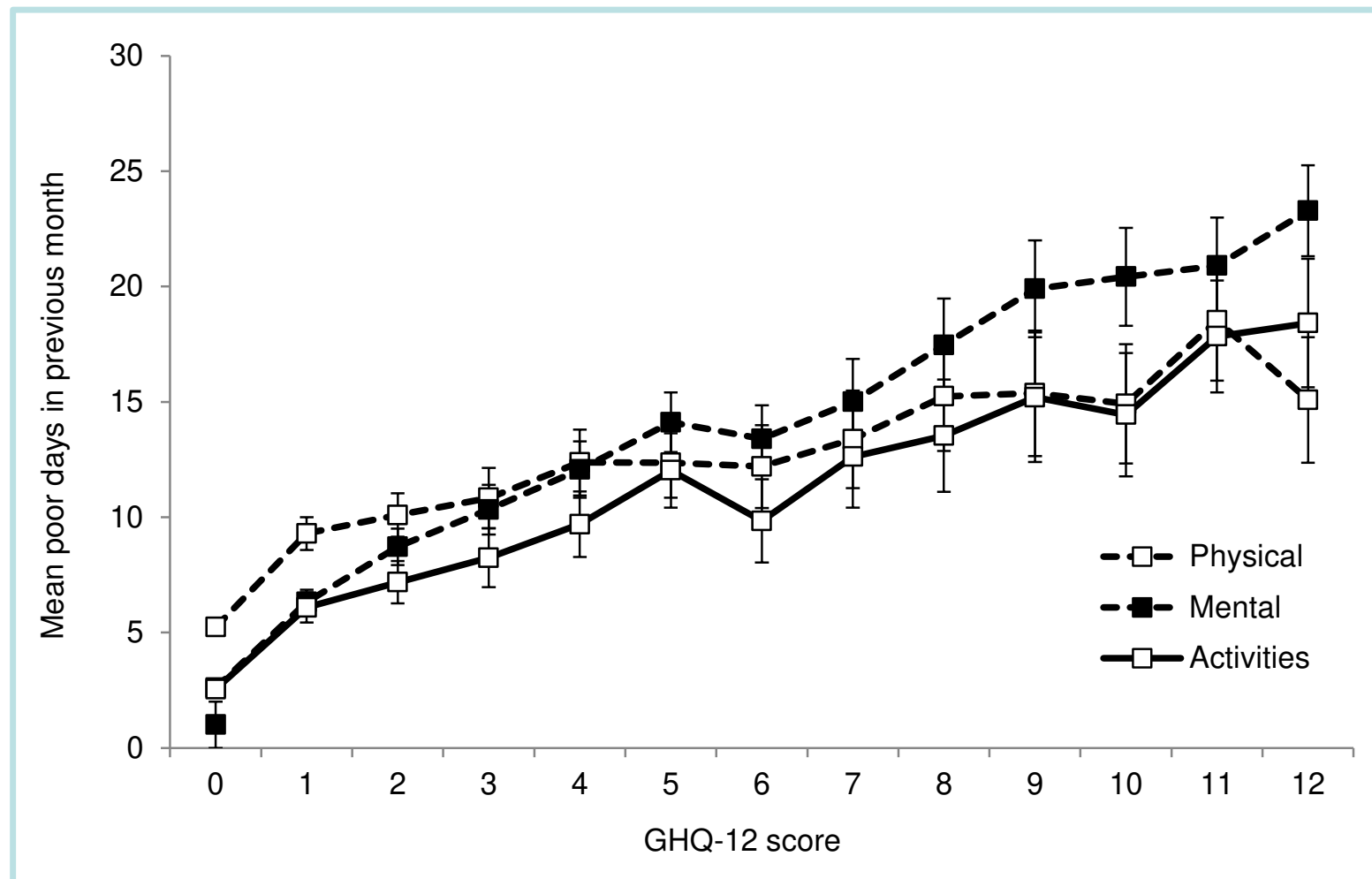

